\title{
The Human RNA Surveillance Factor UPF1 Modulates Gastric Cancer Progression by Targeting Long Non-Coding RNA MALAT1
}

\author{
Li Li $^{a}$ Yingying Geng ${ }^{a}$ Ru Feng ${ }^{a}$ Qinqin Zhu ${ }^{a}$ Bei Miao ${ }^{a}$ Jiang Cao ${ }^{b}$ Sujuan Feia \\ aDepartment of Gastroenterology, 'bepartment of Hematology, the Affiliated Hospital of Xuzhou \\ Medical University, Xuzhou, China
}

\section{Key Words}

Gastric cancer • UPF1 • MALAT1 • Hypermethylation • Nonsense-mediated mRNA decay

\begin{abstract}
Background/Aims: The long non-coding RNA metastasis-associated lung adenocarcinoma transcript 1 (MALAT1) is overexpressed in numerous cancers. However, whether MALAT1 is regulated and the related mechanisms in gastric cancer remain unclear. Methods: Immunohistochemistry and GRT-PCR analyses were used to detect the expression levels of UPF1 and MALAT1 in gastric cancer and adjacent normal tissues. MTT, cell cycle, apoptosis and transwell assays were performed to examine the effects of UPF1 on cell cycle progression, cell proliferation, apoptosis, migration and invasion. Additionally, sodium bisulfate sequencing was used to test the promoter hypermethylation on UPF1 in gastric tumor tissues. Finally, RNA immunoprecipitation and luciferase reporter analyses demonstrated that UPF1 directly bound with MALAT1. Results: The expression of UPF1 was significantly downregulated in gastric cancer and negatively correlated with MALAT1 expression. Patients with lower expression of UPF1 had poorer prognosis than those with higher expression. Overexpression of UPF1 inhibited cell proliferation, cell cycle progression, cell migration and invasion, and promoted cell apoptosis in gastric cancer cells. Moreover, the UPF1-mediated inhibition of gastric cancer progression was reversed by overexpression of MALAT1. A profound downregulation of UPF1 in gastric tumor tissues was due to promoter hypermethylation. Overexpression of UPF1 increased nonsense-mediated mRNA decay (NMD) efficiency and thus led to downregulation of MALAT1. Conclusion: Our results demonstrate that UPF1 is a potential modulator of MALAT1 and that UPF1/MALAT1 pathway could be a therapeutic target for gastric cancer.
\end{abstract}

\section{Introduction}

Gastric cancer is the second leading cause of cancer worldwide [1]. Although the recent advances are acquired in gastric cancer treatment, majority of patients remain incurable

L. Li and Y. Geng contributed equally to this work.

Professor Sujuan Fei or Professor Jiang Cao
Department of Hematology, the Affiliated Hospital of Xuzhou Medical University, No 99, Wset HuaiHai Road, Xuzhou City, Jiangsu Province. 221006, (P.R. China)

E-Mail sujuan_fei@163.com / jiang_cao@yeah.net 


\section{Cellular Physiology Cell Physiol Biochem 2017;42:2194-2206

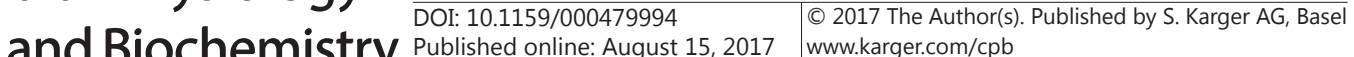 Published online: August 15, 2017 www.karger.com/cpb \\ Li et al.: UPF1 Inhibits Gastric Cancer Progression}

once the metastasis has happened and hold a poor prognosis [2]. However, the detailed mechanisms remain to be elucidated.

Long non-coding RNAs (lncRNAs) are a class of non-coding RNA longer than 200 nucleotides with limited protein coding ability, and act as new modulators in various biological processes, especially in cancers [3,4]. Metastasis associated lung adenocarcinoma transcript-1 (MALAT1), a well-known IncRNA, is significantly upregulated in various cancers, such as non-small cell lung carcinoma (NSCLC) [5], breast cancer [6], hepatocellular carcinoma [7] and gastric cancer [8]. MALAT1 could promote cell proliferation [9], metastasis and invasiveness of gastric adenocarcinoma [10]. However, the mechanisms by which MALAT1 is regulated in gastric cancer are inadequate.

Nonsense-mediated mRNA decay (NMD) pathway is an mRNA surveillance pathway that controls aberrant mRNA transcripts harboring premature termination codons (PTCs), and thus eliminates the synthesis of potential truncated proteins [11]. Importantly, recent study has indicated that NMD is not only a degradation pathway, but also modulates normal gene expression [12]. UPF1 is the core part of human NMD machinery and degrades mRNA transcripts that bind to RNA binding protein Staufen 1 [13]. In addition, UPF1 is essential for accomplishing DNA replication during S phase of the cell cycle [14]. Also, UPF1 is also involved in cell proliferation and differentiation by promoting the proliferative, undifferentiation state or the decay of mRNAs encoding many other proteins that oppose the proliferative, undifferentiated cell state [15]. Another study has shown that UPF1 is down-regulated and commonly mutated in pancreatic adenosquamous carcinoma [16]. However, no studies have investigated the exact activities of UPF1 in human gastric cancer, which promotes us to explore its roles in gastric cancer.

In this study, we showed that UPF1 expression was significantly reduced in gastric cancer tissues compared with the adjacent normal tissues, and negatively correlated with MALAT1 expression. The connection between UPF1 and MALAT1 in gastric cancer tissues promoted us to investigate whether NMD might be involved in the regulation of MALAT1 in gastric cancer. And further functional and mechanistic assays revealed that UPF1 directly binds with MALAT1 and promotes its degradation. Finally, we aimed to indicate that UPF1 overexpression could enhance the chemotherapeutical efficiency for gastric cancer. These results document that NMD is a crucial regulatory event that influences the expression of MALAT1 and UPF1/MALAT1 pathway acts as a way for cells to downregulate the expression of MALAT1. Taken together, our results provide novel insights for ameliorating or finding a new therapeutic target for gastric cancer progression.

\section{Material and Methods}

\section{Gastric clinical samples and cells culture}

38 pairs of gastric tumor and adjacent normal paraffin-embedded tissue samples were randomly selected from the Affiliated Hospital of Xuzhou Medical University between October 2014 and November 2016. The diagnosis of gastric cancer was histopathologically confirmed. 11 patients were diagnosed with stage T, 13 patients were diagnosed with stage $\mathrm{N}$ and 14 patients were diagnosed with stage $\mathrm{M}$. Written informed consent from all patients and approval of the Hospital Ethic Review Committees were obtained. The median follow-up time of the included patients was 8 years and the overall survival was used to compare prognosis and survival. And the mean value was regarded as the cut-off. The human gastric cancer cell lines BGC-823 and SGC-7901 were purchased from the China Academia Sinica Cell Repository (Shanghai). The GES-1 immortalized gastric epithelial cell line and HEK293T cells were purchased from KeyGEN BioTECH (Nanjing, China). All of the gastric cancer cell lines and GES-1 were maintained in RPMI 1640 medium (Gibco), and HEK293T cells were maintained in DMEM medium (Gibco) supplemented with $10 \%$ fetal bovine serum (FBS) (Gibco), $80 \mathrm{U} / \mathrm{ml}$ penicillin and $0.08 \mathrm{mg} / \mathrm{ml}$ streptomycin at $37^{\circ} \mathrm{C}$ under humidified atmosphere with $5 \% \mathrm{CO}_{2}$. 


\section{Cellular Physiology Cell Physiol Biochem 2017;42:2194-2206 \begin{tabular}{l|l|l} 
and Biochemistry $10.1159 / 000479994$ & $\begin{array}{l}\text { () } 2017 \text { The Author(s). Published by S. Karger AG, Basel } \\
\text { www.karger.com/cpb }\end{array}$
\end{tabular} \\ Li et al.: UPF1 Inhibits Gastric Cancer Progression}

Lentivirus plasmids and stable-infected cell lines construction

Lentiviral shRNA targeting human UPF1 was obtained from Sigma (St. Louis, MO). A scramble non-targeting shRNA was used as a control. The shRNA sequences were inserted into pLKO.1. UPF1 and MALAT1 coding sequences were cloned into PLVX-IRES-ZsGreen1, named as Lenti-UPF1 and Lenti-MALAT1, respectively. Lentiviral particles were packaged in HEK293T cells by co-transfecting Lenti-UPF1 or LentiMALAT1, pCMV-dR8.2 and pMD2.G constructs. $48 \mathrm{~h}$ and $72 \mathrm{~h}$ later, supernatants were collected and centrifuged to remove cell debris, and the supernatants were used as virus. For stable-infected cell lines selection, virus supernatants with $2 \mu \mathrm{g} / \mathrm{ml}$ Polybrene were added to BGC-823 and SGC-7901 cells for $72 \mathrm{~h}$. After two rounds infection, the infected cells were selected with puromycin (Sigma, $2 \mu \mathrm{g} / \mathrm{ml}$ for BGC-823 cells and $1.6 \mu \mathrm{g} / \mathrm{ml}$ for SGC-7901 cells) for 2 weeks, followed by qRT-PCR and western blot verification. Fluorescent cell sorting was used to select cells infected with Lenti-UPF1.

3-(4,5-dimethyl-2-thiazolyl)-2,5-diphenyl-2-H-tetrazolium bromide (MTT) assay

MTT (KeyGEN BioTECH) was used to examine cell proliferation. Cells with different treatment were seeded in 96-well plates at 3000-5000 cells/well. After 24, $48 \mathrm{~h}$ and $72 \mathrm{~h}$, the growth rates were determined. Triplicate experiments were performed for each assay.

Cell apoptosis assay

Cell apoptotic rate was evaluated using AnnexinV-FITC and propidium iodide (PI) (Vazyme Biotech, China) with flow cytometry analysis. Cells with different treatment were harvested and washed with icecold PBS. The cells were then double stained with Annexin V-FITC and PI (BD Biosciences, USA) following the manufacturer's protocol. The flow cytometry analysis was conducted by flow cytometry (BD Biosciences, San Jose, CA).

\section{Cell cycle analysis}

Cells with different treatment were harvested and resuspended in $500 \mu \mathrm{l}$ ice-cold $70 \%$ ethanol and stored overnight at $-20^{\circ} \mathrm{C}$. Then the cells were stained by a cell cycle detection kit (KeyGEN BioTECH) following the manufacturer's protocols. Briefly, $100 \mu$ RNaseA was added to each sample and incubated in $37^{\circ} \mathrm{C}$, then cells were stained with PI at $4{ }^{\circ} \mathrm{C}$ for $30 \mathrm{~min}$. Cells were analyzed on a FACScalibur machine (Becton Dickinson) with Cell-Quest software. The percentage of cells in each phase of the cell cycle was analyzed by ModFit LT 4.0 software.

\section{Cell migration and invasion assays}

The migration and invasion of cells were assessed using $8 \mu \mathrm{m}$ chambers with Matrigel-coated or not (BD Biosciences, FranklinLakes, NY, USA). Briefly, cells $\left(8 \times 10^{4}\right)$ were seeded in serum-free medium and were allowed to migrate or invade toward complete media supplemented with $20 \%$ FBS. The cells that invaded through the membrane to the lower surface were fixed in methanol and stained with $0.1 \%$ viola crystalline solution and counted. Six random area from each of the triplicate migration and invasion assays were counted by using phase contrast microscopy. Quantification was done by measuring absorbance value at OD $570 \mathrm{~nm}$ after being destained with glacial acetic acid.

\section{RNA immunoprecipitation (RIP)}

This process was referred to the previous study [17]. Briefly, cells with different treatment were lysed with $25 \mathrm{mM}$ Tris-HCl buffer (pH 7.5) and $100 \mathrm{U} / \mathrm{ml}$ RNase inhibitor (Sigma), and then incubated with protein-A Sepharose beads precoated with $3 \mu \mathrm{g}$ anti-UPF1 antibody or control rabbit IgG for $1.5 \mathrm{~h}$ at $4^{\circ} \mathrm{C}$. The RNA-protein complexes were pulled-down by protein A/G agarose beads and RNA was extracted with Trizol reagent (Invitrogen, USA), followed by detecting MALAT1 with quantitative real-time PCR.

\section{Quantitative real-time PCR ( $q$ RT-PCR)}

Total RNA was prepared from the cells using Trizol reagent following the standard protocols. Then total RNA was reverse transcribed into cDNA with random primer using M-MLV (Promega, USA) following the standard protocols. Afterwards, the mRNA expression levels were detected according to the protocols of SYBR Green master mix (Thermo) and performed on an ABI Prism 7500 Detection System (Applied Bi- 


\section{Cellular Physiology Cell Physiol Biochem 2017;42:2194-2206 \begin{tabular}{c|l|l}
\cline { 2 - 3 } DOI: 10.1159/000479994 & ( 2017 The Author(s). Published by S. Karger AG, Basel
\end{tabular} Published online: August 15, 2017 www.karger.com/cpb \\ Li et al.: UPF1 Inhibits Gastric Cancer Progression}

osystems, Inc., USA). Then, the expression of each transcript was calculated using the $2^{-\Delta \Delta c t}$ method. The primers were described in Table 1 .

\section{mRNA stability assay}

Gastric cancer cells with UPF1 stable expression or not were added with $5 \mu \mathrm{g} / \mathrm{ml}$ of ActD (Sigma) into the medium. Then total RNA was harvested at indicated times and mRNA expression was examined by qRT-PCR. The mRNA half-life was determined by comparing to the mRNA level before adding ActD.

\section{Luciferase Reporter Assay}

MALAT1 promoter sequence was introduced into the pGL3 vector (Promega) for MALAT1 promoter transcriptional activity assay.
Table 1. Primer sequences used for qRT-PCR

\begin{tabular}{ll}
\hline Gene & Sequences (5' to 3') \\
\hline UPF1 of qRT-PCR forward & ACTCTGGTAATGAGGATTTAGTCA \\
UPF1 of qRT-PCR reverse & CCCAACAGCTTGTGGTAGATGTAG \\
MALAT1 of qRT-PCR forward & TGGTAGTGTGTGGTTCTCTTTTGG \\
MALAT1 of qRT-PCR reverse & CTTTTCGCTTTTATTCTGCTTTTG \\
Vimentin of qRT-PCR forward & AGGAACCAATGAGTCCCTGGAACG \\
Vimentin of qRT-PCR reverse & CTGCAGAAAGGCACTTGAAAGCTG \\
E-cadherin of qRT-PCR forward & ATGGCTTCCCTCTTTCATCTCCTG \\
E-cadherin of qRT-PCR reverse & CATAGTTCCGCTCTGTCTTTGGCT \\
Bcl-2 of qRT-PCR forward & GAAAAATAACTTCAAGCAAACATC \\
Bcl-2 of qRT-PCR reverse & AGTAACTCAAAACAAAAGCAAACC \\
Bax of qRT-PCR forward & AGACTCCTCAAGCCTCCTCACCCC \\
Bax of qRT-PCR reverse & GGAAAACGCATTATAGACCACATC \\
GAPDH of qRT-PCR forward & AGGTCGGTGTGAACGGATTTGR \\
GAPDH of qRT-PCR reverse & GGGGTCGTTGATGGCAACA
\end{tabular}

Transfection efficiency was normalized with $\beta$-gal expression.

\section{Sodium bisulfite sequencing}

The CpG islands were analyzed by MethPrimer (http://www.urogene.org/cgi-bin/methprimer/ methprimer.cgi). Firstly, Genomic DNA was extracted using DNeasy Tissue Kit (TIANGEN, China) and then treated with sodium bisulfite using the CpGenome DNA Modification Kit (Millipore) following the manufacturer's protocols. PCR products were subcloned, and constructs with each region from each sample were randomly choosed for sequence analysis. BiQ Analyzer (http://biq-analyzer.bioinf.mpi-inf.mpg.de/.) was used to analyse DNA methylation data.

\section{Western blotting}

Detailed procedure was described elsewhere [18]. The antibodies againstUPF1 (ab109363) E-Cadherin (ab40772), Vimentin (ab8978) and $\beta$-actin (mAbcam 8226) were purchased from Abcam. The antibodies against Bcl-2 (\#4223) and Bax (\#2774) were purchased from Cell Signaling Technology. Chemiluminescence was detected using an enhanced chemiluminescence kit (YI FEI XUE BIO TECH, Nanjing, China) followed by visualizing using the ChemiDoc XRS Detection System (Bio-Rad).

\section{Immunohistochemistry}

The concrete procedures were referred to the previous study [19]. Immunohistochemical stainings of gastric tumors and adjacent normal tissues for UPF1 were performed using an anti-UPF1 antibody (ab109363) and related secondary goat anti-rat antibody (ab7010). DAB staining was used for immunohistochemistry assay. Image-pro Plus software was used to evaluate and then compare the IHCstained tissue. Briefly, Choosing the dye color area (AOI, area of interesting) in the picture and measuring IOD (integrated option density) value of the region and the area of effective statistical area, then measuring the average optical density within the selected area via the IOD/area (density mean) and calculating the average and standard deviation of the same experimental section of each photo. Use statistical methods to analyze the average density of each experimental group and determine whether there are significant differences between the mean values.

Statistical analysis

Significance in expression was determined by Student's t-test. Comparison of categories within a given characteristic was carried out with the $\chi 2$ test, if any of the expected frequencies was less than five, the 


\section{Cellular Physiology Cell Physiol Biochem 2017;42:2194-2206 \begin{tabular}{ll|l} 
and Biochemistry Publisned onlne: August 15, 2017 & $\begin{array}{l}\text { @ } 2017 \text { The Author(s). Published by S. Karger AG, Basel } \\
\text { www.karger.com/cpb }\end{array}$ \\
\hline
\end{tabular}}

Li et al.: UPF1 Inhibits Gastric Cancer Progression

Fisher's exact test was used. Two-sided P-value $<0.05$ was considered statistically significant. Statistical analyses were performed using MATLAB software packages (Mathworks, Natick, MA, USA) and GraphPad Software, Inc.

\section{Results}

UPF1 expression is significantly downregulated in gastric tumor and negatively correlated with MALAT1 expression

Immunohistochemistry staining was performed to detect the expression of UPF1 in human gastric tumor and adjacent normal tissues. UPF1 expression was higher in adjacent normal tissues than in gastric tumor tissues (Fig. 1A and 1B). qRT-PCR was used to further examine the expression levels of UPF1 and MALAT1. As shown in Fig. 1C and 1D, in consistent with the immunohistochemistry results, UPF1 expression levels were significantly increased in normal gastric tissues, while the expression of MALAT1 was decreased. In addition, the expression levels of UPF1 and MALAT were negatively correlated in gastric tumor samples (Fig. 1E). And patients with lower expression of UPF1 have poorer prognosis than those with higher expression (Fig. 1F and 1G). Further in vitro experiments showed that UPF1 expression

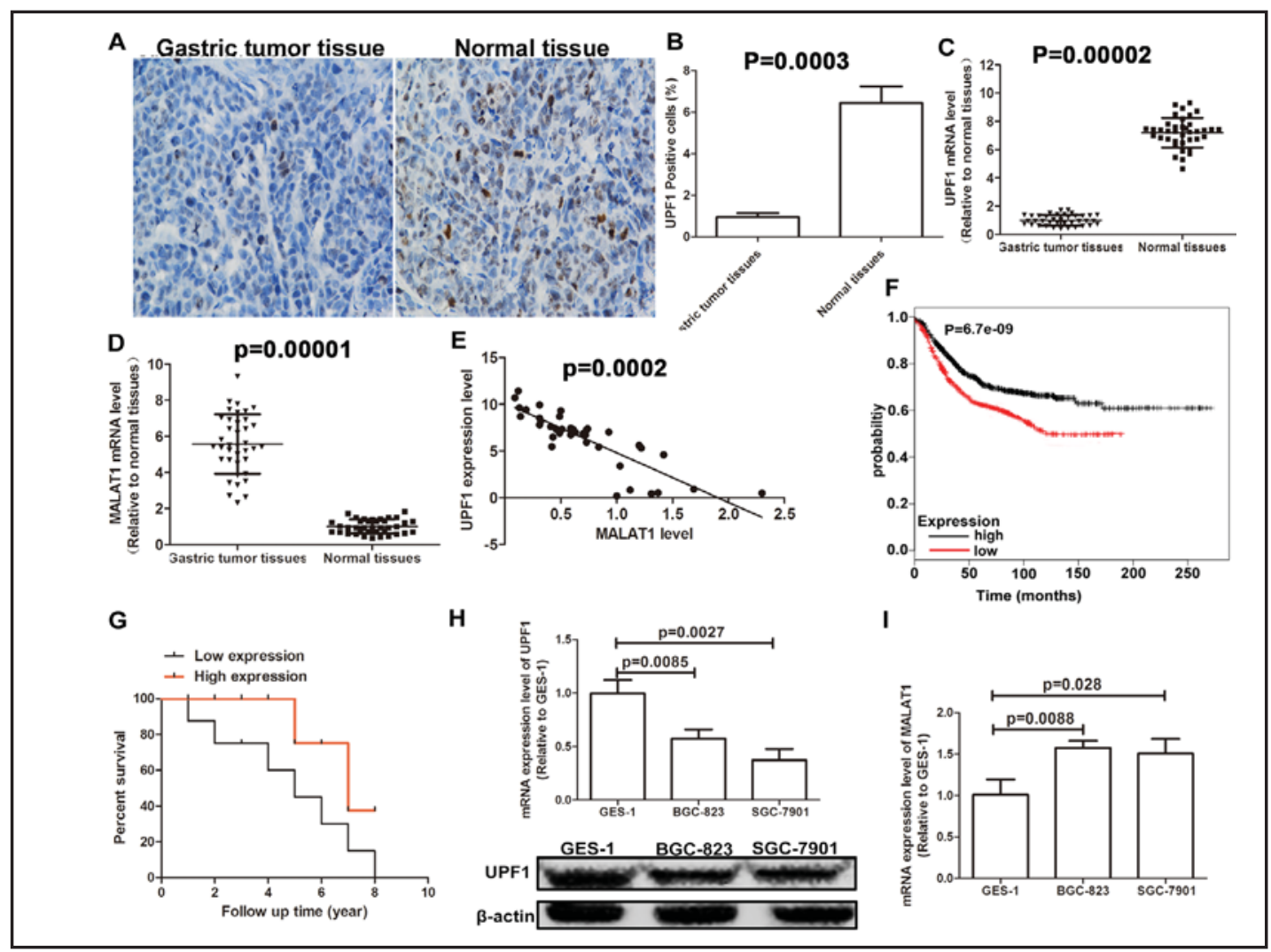

Fig. 1. UPF1 expression is significantly downregulated in gastric tumor and negatively correlated with MALAT1 expression. (A, B) The expression of UPF1 was examined in 38 pairs of gastric tumor and adjacent normal paraffin-embedded tissues via immunohistochemistry analysis. (C, D) The mRNA levels of UPF1 and MALAT1 were examined in 38 pairs of gastric tumor and adjacent normal paraffin-embedded tissues via qRT-PCR analysis. (E) Correlation between the levels of UPF1 and MALAT1. (F, G) Correlation between the levels of UPF1 and the survival of gastric tumor patients. When the expression of UPF1 was considered, patients in the UPF1 low-expression group had a significantly shorter survival time ( $p=6.7 e-09)$. (H) The mRNA and protein levels of UPF1 were examined in gastric cancer cell lines and immortalized gastric epithelial cell line. (I) MALAT1 expression level was detected in gastric cancer cell lines and immortalized gastric epithelial cell line. Data were presented as the mean \pm s.d; ${ }^{* * P}<0.01$ vs. Control. 


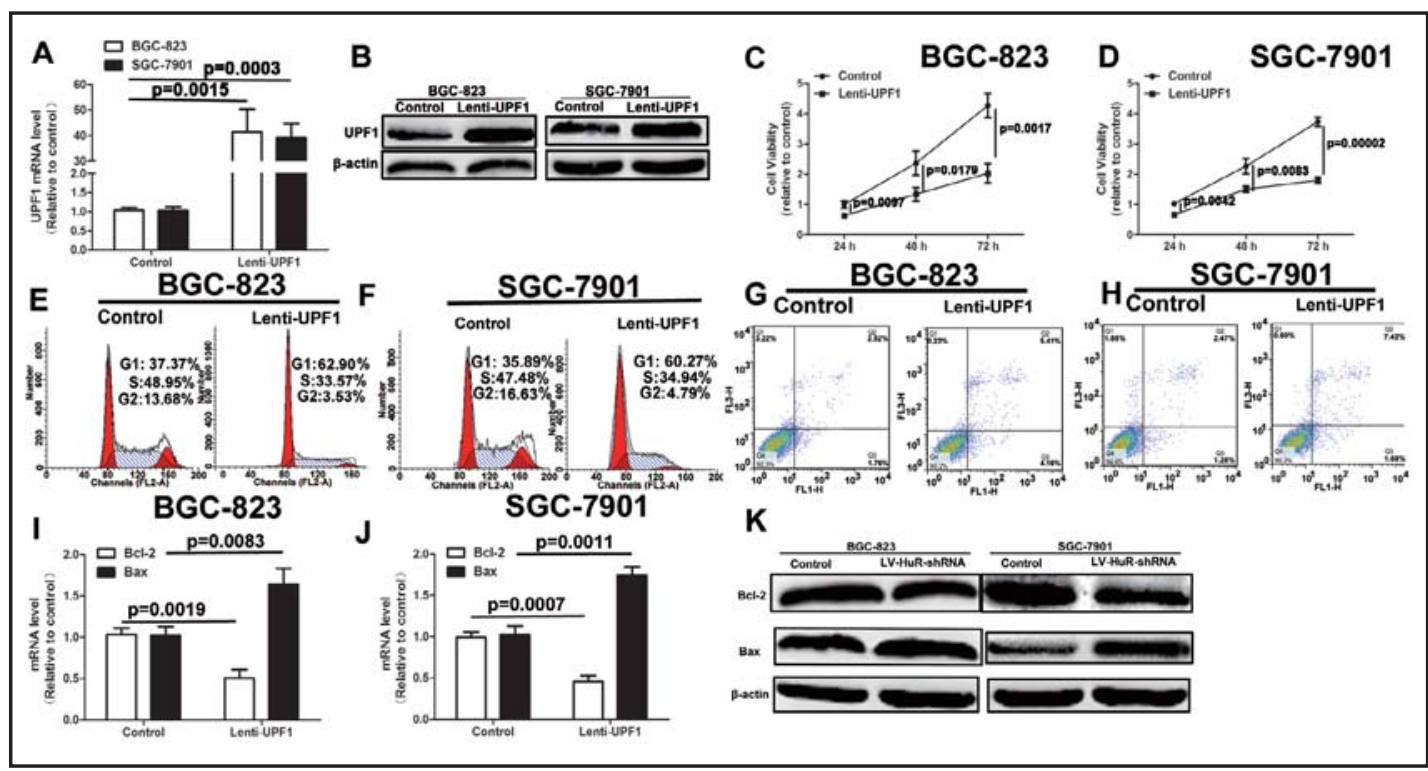

Fig. 2. Overexpression of UPF1 inhibits cell proliferation, cell cycle progression and promotes cell apoptosis in gastric cancer cells. (A, B) UPF1 expression levels were detected in gastric cancer cells with Lenti-UPF1 infection or not. (C, D) The cell viability of BGC-823 and SGC-7901 cells with UPF1 overexpression or not was evaluated by MTT assay. (E, F) Changes in the cell cycle were detected in BGC-823 and SGC-7901 cells with UPF1 overexpression or not. (G, H) Apoptosis was examined in BGC-823 and SGC-7901 cells with UPF1 overexpression or not. (I-K) Apoptotic-related gene (Bcl-2 and Bax) mRNA levels were evaluated in BGC-823 and SGC-7901 cells with UPF1 overexpression or not. Data were presented as the mean \pm s.d; ${ }^{* *} \mathrm{P}<0.01$ vs. Control.

was remarkably decreased in gastric cancer cells compared with that in gastric epithelial cells, while MALAT1 exhibited the opposite results (Fig. 1H and 1I). These results suggest that UPF1 may hold suppressive and MALAT1-related roles in gastric cancer progression.

Overexpression of UPF1 inhibits cell proliferation, cell cycle progression and promotes cell apoptosis in gastric cancer cells

Given the downregulation of UPF1 expression in gastric cancer tissues and cells, we constructed gastric cancer cells with stable overexpression of UPF1 using lentivirus infection. The mRNA and protein levels of UPF1 were remarkably upregulated in BGC-823 and SGC7901 cells with UPF1 stable overexpression (Fig. 2A and 2B). Then MTT assay was conducted to detect the effects of UPF1 on cell proliferation. As shown in Fig. 2C and 2D, overexpression of UPF1 significantly inhibited cell proliferation in BGC-823 and SGC-7901 cells. In addition, cell cycle arrest and apoptotic cells were observed in UPF1 overexpression cells (Fig. 2E$\mathrm{H}$ ). Meanwhile, the mRNA and protein levels of anti-apoptotic protein (Bcl-2) and proapoptotic protein (Bax) were measured by qRT-PCR and western blot. In accordance with the above results, the expression levels of Bcl-2 were downregulated, while Bax expression levels were upregulated in cells with UPF1 overexpression (Fig. 2I-K). Taken together, these results indicate that overexpression of UPF1 could significantly repress gastric cancer cell proliferation, cell cycle progression and promote cell apoptosis.

Overexpression of UPF1 inhibits cell migration, invasion and epithelial-mesenchymal transition (EMT) in gastric cancer cells

Next, the effects of UPF1 on gastric tumor metastasis were further evaluated by transwell migration and invasion assays. As shown in Fig. 3A and 3B, the UPF1 overexpressed cells exhibited a significantly decrease of migration and invasion abilities. As EMT process plays a vital role in tumor metastasis, the effects of UPF1 on EMT were further examined in gastric cancer cells. As expected, overexpression of UPF1 suppressed the expression of 


\section{Cellular Physiology Cell Physiol Biochem 2017;42:2194-2206

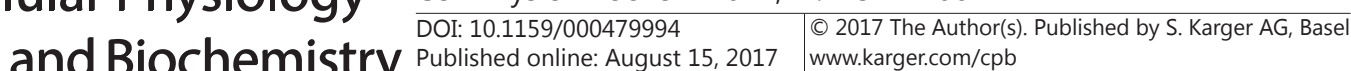

Li et al.: UPF1 Inhibits Gastric Cancer Progression
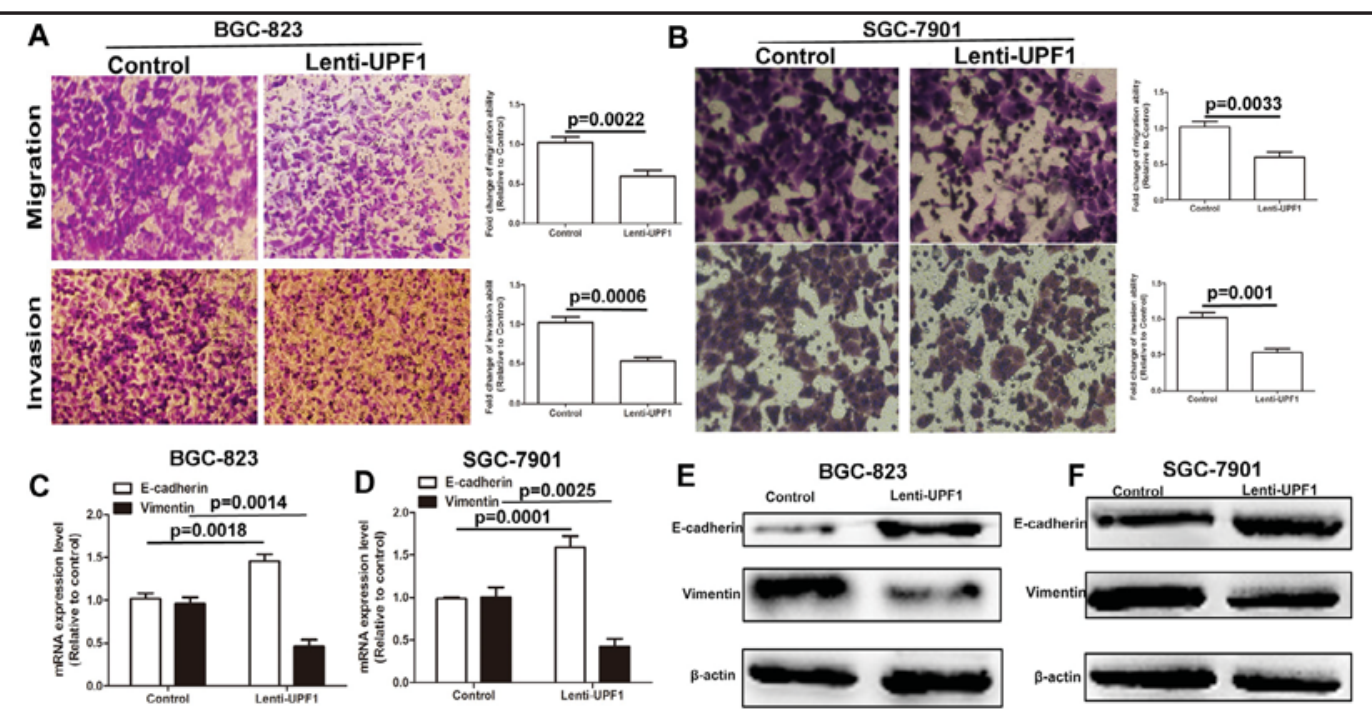

Fig. 3. Overexpression of UPF1 inhibits cell migration, invasion and epithelial-mesenchymal transition (EMT) in gastric cancer cells. (A, B) Overexpression of UPF1 inhibited cellular migration in BGC-823 and SGC-7901 cells. (C-F) Overexpression of UPF1 blocked EMT process characterized as upregulation of epithelial marker E-cadherin expression and downregulation of mesenchymal marker Vimentin expression. Data were presented as the mean \pm s.d; ${ }^{* *} \mathrm{P}<0.01$ vs. Control.

mesenchymal-related protein (vimentin), but increased the expression of epithelial-related protein (E-cadherin) at both mRNA and protein levels in BGC-823 and SGC-7901 cells (Fig. 3C-F). Accordingly, our results demonstrate that UPF1 overexpression inhibits cell metastasis and EMT in gastric cancer.

\section{CPG hypermethylation downregulates UPF1 expression in gastric cancer}

Previous study has verified that hypermethylation of tumor suppressor genes contributes to gastric tumorigenesis [20] and UPF1 hypermethylation has been shown in hepatocellular carcinoma [21]. To investigate the underlying mechanism responsible for UPF1 downregulation in gastric tumor, we detected whether hypermethylation was involved in the progression. Several CpG islands were shown in promoter region of UPF1 with MethPrimer software assay. Thus, we speculated that hypermethylation may take a critical role in regulating of UPF1 expression. As shown in Fig. 4A, the mRNA and protein expression levels of UPF1 were gradually increased in BGC-823 and SGC-7901 cells following treatment with increased concentrations of 5-Aza-2'-deoxycytidine which is a methylation inhibitor and can demethylate genomic DNA. To further explore the relationship between promoter methylation and UPF1 gene downregulation, CpG island prediction software was used to analyze the promoter region of UPF1. And we found that the CpG island was enriched in the a putative promoter region of UPF1 (Fig. 4B). Furthermore, the fragment containing CpG dinucleotides was amplified from genomic DNA isolated from gastric tumor and normal adjacent tissues treated with either sodium bisulfite or vehicle. Fig. 4C indicated a representative methylation pattern of the $\mathrm{CpGs}$ putative promoter region, and the majority of CpGs were unmethylated in normal tissues, whereas most of the CpGs were methylated in the tumor tissues.

UPF1 suppresses gastric tumorigenesis by directly binding MALAT1

As UPF1 serves as an important factor in surveillance pathway which could target and thus downreuglate aberrant genes expression in tumors [22]. Whether UPF1 affects MALAT1 expression in gastric tumor progression? This promoted us to investigate the relationship between UPF1 and MALAT1 in gastric cancer. qRT-PCR analysis showed that MALAT1 

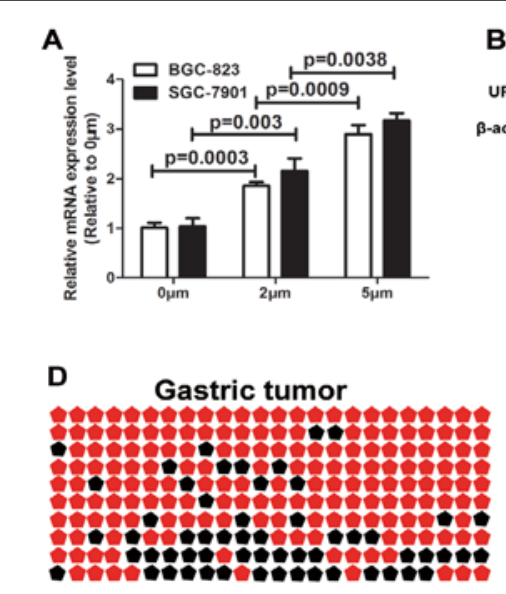
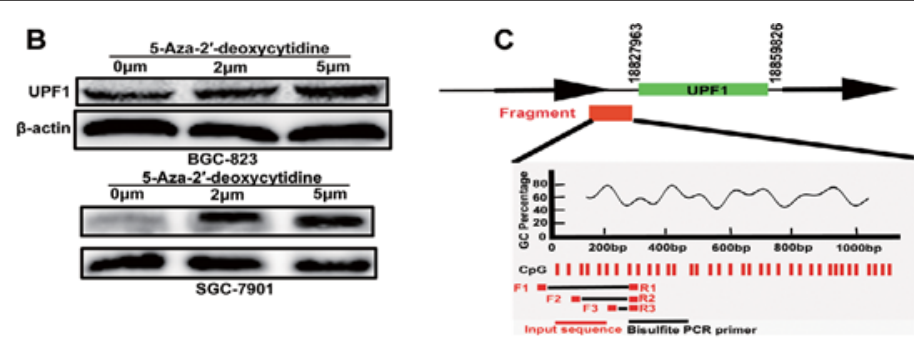

Normal adjacent tissue

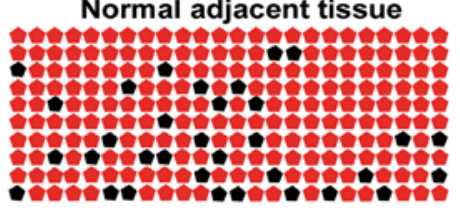

Fig. 4. $\mathrm{CpG}$ hypermethylation downregulates UPF1 expression in gastric cancer. (A, B) Demethylation following treatment with 5-Aza-2' -deoxycytidine induced UPF1 up-regulation in BGC-823 and SGC-7901 cells, the protein and mRNA expression levels of UPF1 were checked by qRT-PCR and western blot respectively. (C) The CpG island enriched sites in the upstream region of UPF1 was analyzed using CpG islandsearcher. (D) Bisulfite sequencing of the putative promoter region. Each line represents one PCR product, and ten PCR products are shown for each sample., ,methylatedCpGs;, unmethylated CpGs. Values are the mean \pm SD. ${ }^{* *} \mathrm{P}<0.01$.

expression level was downregulated in cells with UPF1 overexpression in BGC-823 and SGC7901 (Fig. 5A), whereas UPF1 knockdown in GES-1 cells increased MALAT1 expression (Fig. 5B). The mRNA stability of MALAT1 was further tested in gastric tumor cells with UPF1 overexpression and gastric epithelial cells with UPF1 knockdown. As expected, the decay rate of MALAT1 was increased in in BGC-823 and SGC-7901 cells with UPF1 overexpression $\left(\mathrm{t}_{1 / 2}=7.4 \pm 0.3 \mathrm{~h}\right.$ vs $\mathrm{t}_{1 / 2}=5.4 \pm 0.2 \mathrm{~h}$ in BGC-823 cells and $\mathrm{t}_{1 / 2}=5.7 \pm 0.2 \mathrm{~h}$ vs $\mathrm{t}_{1 / 2}=3.8 \pm 0.2 \mathrm{~h}$ in SGC-7901 cells), but decreased in GES-1 cells with UPF1 knockdown $\left(\mathrm{t}_{1 / 2}=4.8 \pm 0.2 \mathrm{~h}\right.$ vs $\mathrm{t}_{1 / 2}=7.9 \pm 0.4 \mathrm{~h}$ ) (Fig. 5C-E). However, the luciferase reporter assay showed that MALAT1 promoter transcriptional activity was not affected by UPF1 (Fig. 5F), demonstrating that UPF1 could not alter the transcriptional activity of MALAT1. In addition, to investigate whether UPF1 bound to MALAT1, UPF1-binding complex with UPF1 antibody in BGC-823 and SGC-7901 cells with UPF1 overexpression was pull down, followed by examination the bound mRNAs by qRT-PCR. As shown in Fig. 5G and 5H, RIP assays indicated that the UPF1 associated with MALAT1 more specifically than control. Therefore, these results indicated that UPF1 interacts and stabilizes MALAT1. We further investigated whether UPF1 inhibited gastric tumor progression in a MALAT1-dependent way. Overexpression of MALAT1 attenuated or even reversed UPF1-mediated inhibitions on gastric tumor cell proliferation, EMT and the promotion on cell apoptosis (Fig. 5I-L). Therefore, these results indicate that UPF1 interacts and inhibits MALAT1 expression, and thus exerts inhibitory effects on gastric tumor progression in a MALAT1-dependent way.

Overexpression of UPF1 enhances chemotherapeutical sensitivity in gastric cancer cells

We further explored whether ectopic expression of UPF 1 affects the chemotherapeutical sensitivity of gastric cancer cells. MTT assays showed that BGC-823 and SGC-7901 cells with UPF1 overexpression displayed high doxorubicin sensitivity (Fig. 6A). Meanwhile, when treated with doxorubicin, BGC-823 and SGC-7901 cells showed a decrease of cells in S phase, while BGC-823 and SGC-7901 cells with UPF1 overexpression showed a higher change (Fig. 6B). In addition, upregulating UPF1 in BGC-823 and SGC-7901 cells promoted apoptosis induced by doxorubicin treatment (Fig. 6C). The protein levels of apoptotic markers (Bcl2 and Bax) were examined by western blot. As shown in Fig. 6D, exposure of cells with 


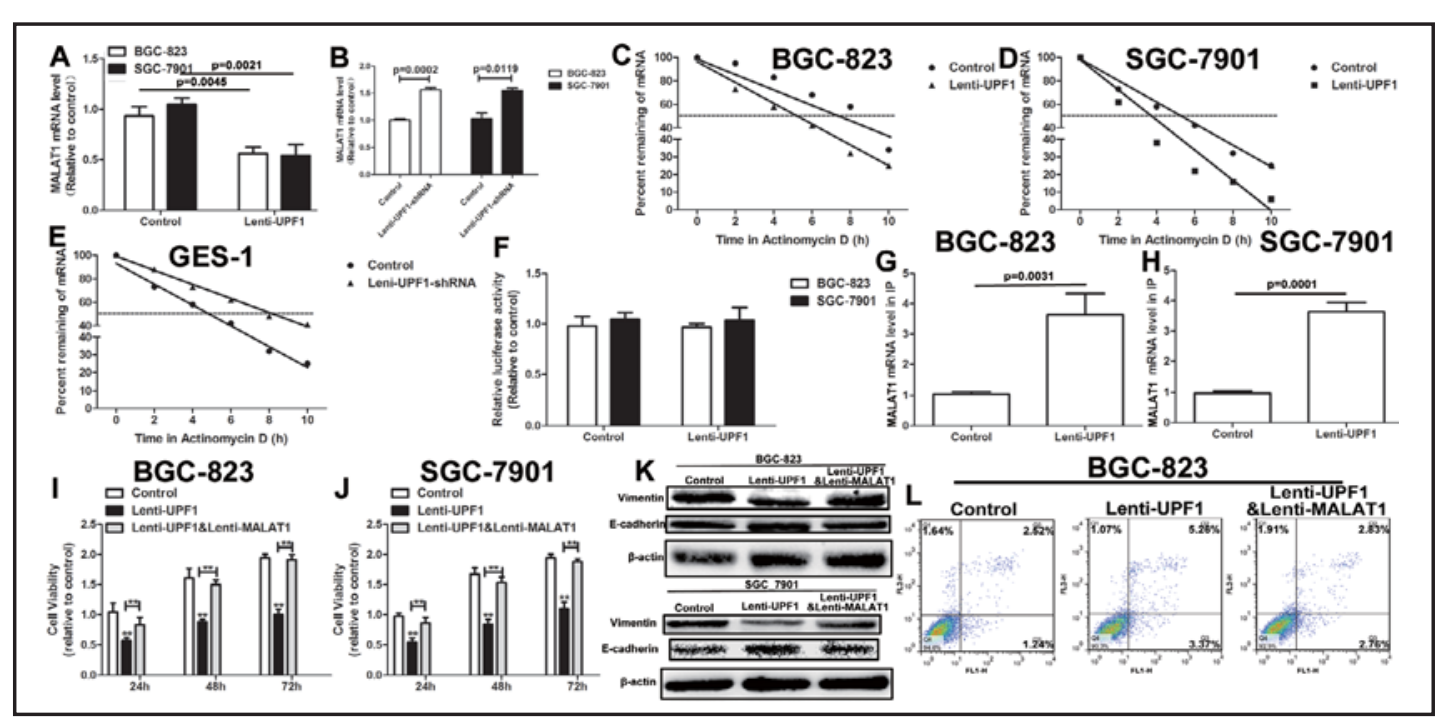

Fig. 5. UPF1 suppressed gastric tumorigenesis by directly binding MALAT1. (A) MALAT1 expression was detected in BGC-823 and SGC-7901 cells with UPF1 overexpression by qRT-PCR. (B) MALAT1 expression was detected in GES-1 cells with UPF1 knockdown by qRT-PCR. (C, D) Cells depicted in (A) were treated with actinomycin D $(2.5 \mu \mathrm{g} / \mathrm{ml})$ for the indicated times. MALAT1 mRNA level was measured by qRT-PCR and the percentage of mRNA that remained was plotted. (E) Cells depicted in (B) were treated with actinomycin $\mathrm{D}(2.5 \mu \mathrm{g} / \mathrm{ml})$ for the indicated times. MALAT1 mRNA level was measured by qRT-PCR and the percentage of mRNA that remained was plotted. (F) Ectopic UPF1 expression did not affect the promoter activity of MALAT1. (G, H) qRT-PCR was used to measure the abundance of MALAT1 mRNA present in the Ago2-IP materials after the RIP assay in BGC-823 and SGC-7901 cells infected with Lenti-UPF1. (I, J) Cell viability was detected in SCC25 and FaDu cells with different treatments by MTT assay as indicated. (K) The EMT markers (E-cadherin and Vimentin) expression was determined as indicated. (L) BGC-823 cells apoptosis was examined with Lenti-UPF1 infection plus Lenti-MALAT1 infection or nor. Data were presented as the mean \pm s.d; ${ }^{* *} \mathrm{P}<0.01$ vs. Control.

UPF1 overexpression to doxorubicin increased the expression of Bax and decreased Bcl-2 expression. Hence, these results indicate that enforced expression of UPF1 could enhance doxorubicin sensitivity in BGC-823 and SGC-7901 cells.

\section{Discussion}

Here, we reported the correlation between UPF1 downregulation and gastric tumors. Immunohistochemical analysis, western blot and qRT-PCR analyses showed that the downregulation of UPF1 expression was associated with malignant progression of gastric tumor. Epigenetic silencing of UPF1 gene was observed in gastric tumor cell lines. In addition, we confirmed that UPF1 could bind with IncRNA MALAT1 directly and decreased MALAT1 expression in gastric cancer cells. And UPF1 exerted its inhibitory effects on gastric tumor progression in a MALAT1-dependent manner. Notably, we demonstrated that enforced expression of UPF1 could enhance doxorubicin sensitivity in BGC-823 and SGC7901 cells. Therefore, we strongly believed that UPF1 downregulation may be required for gastric tumorigenesis and has a potential to be developed as a biomarker for gastric tumor. However, it still needs reasonable clinical trials for evaluation of this biomarker for future application.

Previous studies have shown that demethylation of oncogenes or hypermethylation of tumor suppressors contributed to tumorigenesis [20,23]. Tumor-related hypomethylation 


\section{Cellular Physiology Cell Physiol Biochem 2017;42:2194-2206 \begin{tabular}{c|l|l} 
DOI: 10.1159/000479994 & ( ) 2017 The Author(s). Published by S. Karger AG, Basel
\end{tabular}}

Li et al.: UPF1 Inhibits Gastric Cancer Progression

Fig. 6. Overexpression of UPF1 enhances chemotherapeutical sensitivity in gastric cancer cells. (A, B) BGC-823 and SGC-7901 cells were infected with Lenti-UPF1 followed by the treatment of doxorubicin, then the cell viability was examined by MTT assay. (C) BGC-823 and SGC-7901 cells were infected with Lenti-UPF1 followed by the treatment of doxorubicin, then the cell cycle was examined. (D) BGC-823 and SGC-7901 cells were infected with Lenti-UPF1 followed by the treatment of doxorubicin, then the cell apoptosis was measured. (E, F) The crucial marker of cell apoptosis (Bcl-2 and Bax) protein levels were evaluated in cells depicted in (A, B). Data were presented as the mean \pm s.d; ** $\mathrm{P}<0.01$ vs. Control.

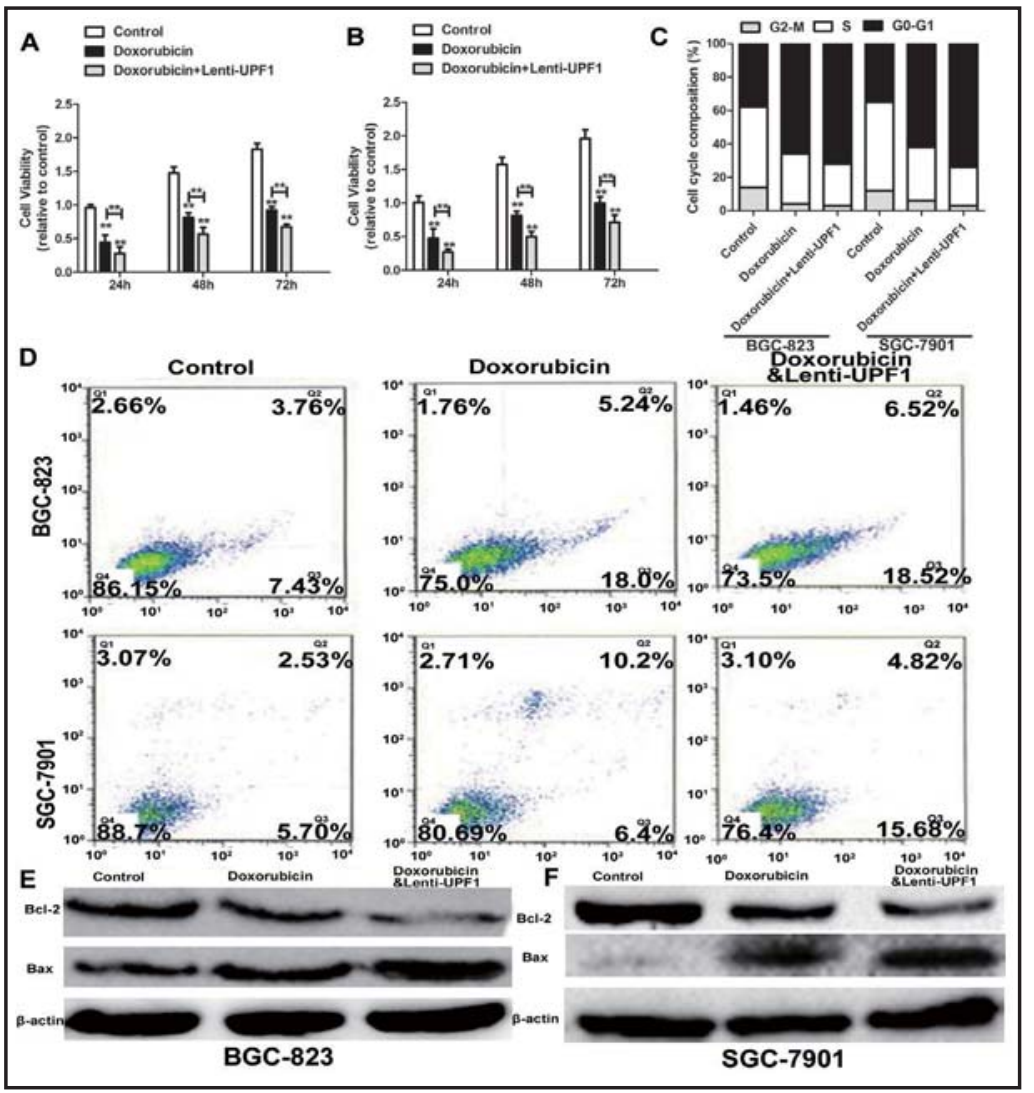

of gene promoter is often linked with aberrant cell proliferation [24, 25]. Our results showed that UPF1 is a potential suppressor in gastric tumor, which holds inhibitory effects on gastric cancer cell proliferation, cell cycle progression and migration. Importantly, promoter region was found to be hypermethylated and treatment with 5-Ad reversed the expression of UPF1, demonstrating that UPF1 expression was also affected by the status of DNA demethylation.

Over the past decades, the functional characterization of genes has primarily focused on the role of coding genes and lncRNAs are generally considered as non-functional genes which could not code proteins or code truncated proteins [26, 27]. Indeed, the recent explosion in knowledge demonstrating the importance of lncRNAs in diseases, especially in cancer, has brought these heretofore neglected molecules to the forefront. Numerous studies have indicated that IncRNAs play important roles in tumor development. For examples, IncRNA MALAT1 could induced migration and invasion of human breast cancer cells by competitively binding miR-1 with cdc42 [28]. MALAT1-derived mascRNA is involved in cardiovascular innate immunity [29]. And downregulation of MALAT1 affects proliferation and the expression of stemness markers in glioma stem cell line SHG139S [30]. In addition, the potential employment of IncRNAs as novel biomarkers is supported by widespread observations [31]. Previous study has indicated the promotive roles of MALAT1 in gastric tumor [9], here we further confirmed this idea and showed that overexpression of MALAT1 could attenuate or even reverse the inhibitory effects of UPF1 on gastric tumor. Taken together, it is becoming clear that insight into the widespread influences exerted by MALAT1 may help us identify novel biomarkers for gastric cancer diagnosis and present new opportunities for MALAT1-based targeted therapy.

To prevent the accumulation of truncated proteins, aberrant mRNAs containing premature termination codons could be degraded by NMD, which is an evolutionally mechanism controlling mRNA quality [32]. UPF1, UPF2 and UPF3 make up the NMD 


\section{Cellular Physiology Cell Physiol Biochem 2017;42:2194-2206 \begin{tabular}{ll|l}
\cline { 2 - 2 } and Biochemistry & Dublished on/ne: August 15, 2017 & $\begin{array}{l}\text { @ } 2017 \text { The Author(s). Published by S. Karger AG, Basel } \\
\text { www.karger.com/cpb }\end{array}$ \\
\hline
\end{tabular} Li et al.: UPF1 Inhibits Gastric Cancer Progression}

machinery and UPF1 is a critical member in this protein set. UPF1 works together with eRF1/ eRF3 to distinguish aberrant translation termination events and, in concert with UPF2 and UPF3, triggers mRNA degradation [33]. Upf1-bound transcripts support the contribution of NMD to epileptogenesis [34]. More importantly, UPF1 also modulates RNA transcripts in a NMD-independent way. For example, UPF1 regulates tumorigenesis by targeting Smad7 in hepatocellular carcinoma [21]. And UPF1 regulates myeloid cell functions and S100A9 expression by the hnRNP E2/miRNA-328 balance [35]. In this study, we found that UPF1 was also downregulated in gastric cancer. Notably, we showed that UPF1 could promote MALAT1 mRNA degradation and thus inhibit MALAT1 expression in gastric cancer. However, in the present study, we did not investigate the detailed binding sites of UPF1 in MALAT1, which should be explored in the following studies, and the future researches should be performed to confirm whether the UPF1/MALAT1 pathway did exist in vivo and other tumors.

All together, our results indicated that UPF1 played an important role during gastric carcinogenesis and may serve as a putative target for gastric cancer diagnosis and therapy.

\section{Acknowledgements}

This work was supported in part by grants from the National Natural Science Foundation of China (grant no. 81470303); the Jiangsu Province Natural Science Foundation of China (grant no. BK20141140); University Natural Science Research Project of the Jiangsu Province (grant no. 14KJB320021).

\section{Disclosure Statement}

The authors declare that they have no conflict of interests.

\section{References}

1 Nobili S, Bruno L, Landini I, Napoli C, Bechi P, Tonelli F, Rubio CA, Mini E, Nesi G: Genomic and genetic alterations influence the progression of gastric cancer. World J Gastroenterol 2011;17:290-299.

2 Gao LM: The book gastric cancer: Diagnosis and treatment of gastric cancer. Transl Gastroenterol Hepatol 2016;1:54.

3 Wang L, Chen Z, An L, Wang Y, Zhang Z, Guo Y, Liu C: Analysis of long non-coding rna expression profiles in non-small cell lung cancer. Cell Physiol Biochem 2016;38:2389-2400.

4 Wang X, Li T, Li M, Cao N, Han J: The functional socs3 rs115785973 variant regulated by mir-4308 promotes gastric cancer development in chinese population. Cell Physiol Biochem 2016;38:1796-1802.

-5 Gutschner T, Hammerle M, Eissmann M, Hsu J, Kim Y, Hung G, Revenko A, Arun G, Stentrup M, Gross M, Zornig M, MacLeod AR, Spector DL, Diederichs S: The noncoding rna malat1 is a critical regulator of the metastasis phenotype of lung cancer cells. Cancer Res 2013;73:1180-1189.

6 Meseure D, Vacher S, Lallemand F, Alsibai KD, Hatem R, Chemlali W, Nicolas A, De Koning L, Pasmant E, Callens C, Lidereau R, Morillon A, Bieche I: Prognostic value of a newly identified malat1 alternatively spliced transcript in breast cancer. Br J Cancer 2016;114:1395-1404.

7 Malakar P, Shilo A, Mogilavsky A, Stein I, Pikarsky E, Nevo Y, Benyamini H, Elgavish S, Zong X, Prasanth KV, Karni R: Long noncoding rna malat1 promotes hepatocellular carcinoma development by srsf1 upregulation and mtor activation. Cancer Res 2016

8 Lee NK, Lee JH, Ivan C, Ling H, Zhang X, Park CH, Calin GA, Lee SK: Malat1 promoted invasiveness of gastric adenocarcinoma. BMC Cancer 2017;17:46.

-9 Wang J, Su L, Chen X, Li P, Cai Q, Yu B, Liu B, Wu W, Zhu Z: Malat1 promotes cell proliferation in gastric cancer by recruiting sf2/asf. Biomed Pharmacother 2014;68:557-564. 


\section{Cellular Physiology Cell Physiol Biochem 2017;42:2194-2206 \begin{tabular}{ll|l} 
DOI: 10.1159/000479994 & $\begin{array}{l}\text { ○ } 2017 \text { The Author(s). Published by S. Karger AG, Basel } \\
\text { www.karger.com/cpb }\end{array}$ \\
\hline
\end{tabular}}

Li et al.: UPF1 Inhibits Gastric Cancer Progression

10 Qi Y, Ooi HS, Wu J, Chen J, Zhang X, Tan S, Yu Q Li YY, Kang Y, Li H, Xiong Z, Zhu T, Liu B, Shao Z, Zhao X: Malat1 long ncrna promotes gastric cancer metastasis by suppressing pcdh10. Oncotarget 2016;7:1269312703.

-11 Rodriguez-Gabriel MA, Watt S, Bahler J, Russell P: Upf1, an rna helicase required for nonsense-mediated mrna decay, modulates the transcriptional response to oxidative stress in fission yeast. Mol Cell Biol 2006;26:6347-6356.

12 Schweingruber C, Rufener SC, Zund D, Yamashita A, Muhlemann O: Nonsense-mediated mrna decay mechanisms of substrate mrna recognition and degradation in mammalian cells. Biochim Biophys Acta 2013;1829:612-623.

13 Kim YK, Furic L, Desgroseillers L, Maquat LE: Mammalian staufen1 recruits upf1 to specific mrna 3'utrs so as to elicit mrna decay. Cell 2005;120:195-208.

14 Azzalin CM, Lingner J: The human rna surveillance factor upf1 is required for s phase progression and genome stability. Curr Biol 2006;16:433-439.

-15 Lou CH, Shao A, Shum EY, Espinoza JL, Huang L, Karam R, Wilkinson MF: Posttranscriptional control of the stem cell and neurogenic programs by the nonsense-mediated rna decay pathway. Cell Rep 2014;6:748764.

16 Liu C, Karam R, Zhou Y, Su F, Ji Y, Li G, Xu G, Lu L, Wang C, Song M, Zhu J, Wang Y, Zhao Y, Foo WC, Zuo M, Valasek MA, Javle M, Wilkinson MF, Lu Y: The upf1 rna surveillance gene is commonly mutated in pancreatic adenosquamous carcinoma. Nat Med 2014;20:596-598.

-17 Xu CZ, Jiang C, Wu Q, Liu L, Yan X, Shi R: A feed-forward regulatory loop between hur and the long noncoding rna hotair promotes head and neck squamous cell carcinoma progression and metastasis. Cell Physiol Biochem 2016;40:1039-1051.

18 Zheng L, Li X, Meng X, Chou J, Hu J, Zhang F, Zhang Z, Xing Y, Liu Y, Xi T: Competing endogenous rna networks of cyp4z1 and pseudogene cyp4z2p confer tamoxifen resistance in breast cancer. Mol Cell Biol 2016;427:133-142.

19 Li X, Zheng L, Zhang F, Hu J, Chou J, Liu Y, Xing Y, Xi T: Stard13-correlated cerna network inhibits emt and metastasis of breast cancer. Oncotarget 2016;7:23197-23211.

20 Vogiatzi P, Vindigni C, Roviello F, Renieri A, Giordano A: Deciphering the underlying genetic and epigenetic events leading to gastric carcinogenesis. J Cell Physiol 2007;211:287-295.

21 Chang L, Li C, Guo T, Wang H, Ma W, Yuan Y, Liu Q, Ye Q, Liu Z: The human rna surveillance factor upf1 regulates tumorigenesis by targeting smad7 in hepatocellular carcinoma. J Exp Clin Cancer Res 2016;35:8.

22 Karam R, Carvalho J, Bruno I, Graziadio C, Senz J, Huntsman D, Carneiro F, Seruca R, Wilkinson MF, Oliveira $\mathrm{C}$ : The nmd mrna surveillance pathway downregulates aberrant e-cadherin transcripts in gastric cancer cells and in cdh1 mutation carriers. Oncogene 2008;27:4255-4260.

23 Cadieux B, Ching TT, VandenBerg SR, Costello JF: Genome-wide hypomethylation in human glioblastomas associated with specific copy number alteration, methylenetetrahydrofolate reductase allele status, and increased proliferation. Cancer Res 2006;66:8469-8476.

24 Shi M, Wang S, Yao Y, Li Y, Zhang H, Han F, Nie H, Su J, Wang Z, Yue L, Cao J, Li Y: Biological and clinical significance of epigenetic silencing of marveld1 gene in lung cancer. Sci Rep 2014;4:7545.

25 Chen Q, Qin R, Fang Y, Li H, Liu Y: A functional variant at the mir-214 binding site in the methylenetetrahydrofolatereductase gene alters susceptibility to gastric cancer in a chinese han population. Cell Physiol Biochem 2015;36:622-630.

26 Akerman I, Tu Z, Beucher A, Rolando DM, Sauty-Colace C, Benazra M, Nakic N, Yang J, Wang H, Pasquali L, Moran I, Garcia-Hurtado J, Castro N, Gonzalez-Franco R, Stewart AF, Bonner C, Piemonti L, Berney T, Groop L, Kerr-Conte J, Pattou F, Argmann C, Schadt E, Ravassard P, Ferrer J: Human pancreatic beta cell lncrnas control cell-specific regulatory networks. Cell Metab 2017;25:400-411.

27 Jiang H, Wang H, Ge F, Wu L, Wang X, Chen S: The functional variant in the 3'utr of igf1 with the risk of gastric cancer in a chinese population. Cell Physiol Biochem 2015;36:884-892.

28 Chou J, Wang B, Zheng T, Li X, Zheng L, Hu J, Zhang Y, Xing Y, Xi T: Malat1 induced migration and invasion of human breast cancer cells by competitively binding mir-1 with cdc42. Biochem Biophys Res Commun 2016;472:262-269. 

Cellular Physiology Cell Physiol Biochem 2017;42:2194-2206
\begin{tabular}{l|l|l|l|}
\hline DOI: 10.1159/000479994 & 2017 The Author(s). Published by S. Karger AG, Basel \\
\hline
\end{tabular} and Biochemistry Published online: August 15, 2017 www.karger.com/cpb

Li et al.: UPF1 Inhibits Gastric Cancer Progression

29 Gast M, Schroen B, Voigt A, Haas J, Kuehl U, Lassner D, Skurk C, Escher F, Wang X, Kratzer A, Michalik K, Papageorgiou A, Peters T, Loebel M, Wilk S, Althof N, Prasanth KV, Katus H, Meder B, Nakagawa S, Scheibenbogen C, Schultheiss HP, Landmesser U, Dimmeler S, Heymans S, Poller W: Long noncoding rna malat1-derived mascrna is involved in cardiovascular innate immunity. J Mol Cell Biol 2016;8:178-181.

30 Han Y, Zhou L, Wu T, Huang Y, Cheng Z, Li X, Sun T, Zhou Y, Du Z: Downregulation of Incrna-malat1 affects proliferation and the expression of stemness markers in glioma stem cell line shg139s. Cell Mol Neurobiol 2016;36:1097-1107.

-31 Han Y, Wu Z, Wu T, Huang Y, Cheng Z, Li X, Sun T, Xie X, Zhou Y, Du Z: Tumor-suppressive function of long noncoding rna malat1 in glioma cells by downregulation of mmp2 and inactivation of erk/mapk signaling. Cell Death Dis 2016;7:e2123.

-32 Azzalin CM, Lingner J: The double life of upf1 in rna and DNA stability pathways. Cell Cycle 2006;5:14961498.

33 Cheng Z, Morisawa G, Song H: Biochemical characterization of human upf1 helicase. Methods Mol Biol 2010;587:327-338.

34 Mooney CM, Jimenez-Mateos EM, Engel T, Mooney C, Diviney M, Veno MT, Kjems J, Farrell MA, O'Brien DF, Delanty N, Henshall DC: Rna sequencing of synaptic and cytoplasmic upf1-bound transcripts supports contribution of nonsense-mediated decay to epileptogenesis. Sci Rep 2017;7:41517.

-35 Saul MJ, Stein S, Grez M, Jakobsson PJ, Steinhilber D, Suess B: Upf1 regulates myeloid cell functions and s100a9 expression by the hnrnp e2/mirna-328 balance. Sci Rep 2016;6:31995. 